\title{
DOES RETROGRADE WARM BLOOD CARDIOPLEGIC PERFUSION PROVIDE BETTER PROTECTION OF ISCHEMIC AREAS THAN ANTEGRADE WARM BLOOD CARDIOPLEGIC PERFUSION? A MAGNETIC RESONANCE STUDY IN PIG HEARTS
}

Jian Ye, MD

Jiankang Sun, MSc ${ }^{\mathrm{a}}$

Edward F. Hoffenberg, MSc ${ }^{a}$

Jie Shen, $\mathrm{PhD}^{\mathrm{a}}$

Luojia Yang, MD

Randy Summers, $\mathrm{MSc}^{\mathrm{a}}$

Tomas A. Sálerno, $\mathrm{MD}^{\mathrm{b}}$

Roxanne Deslauriers, $\mathrm{PhD}^{\mathrm{a}}$
Objective: The purpose of this study was to determine whether retrograde continuous normothermic blood cardioplegic perfusion provides better protection to ischemic areas of the left and right ventricles than does antegrade continuous normothermic blood cardioplegic perfusion. Localized phosphorus 31 magnetic resonance spectroscopy was used to monitor the changes in energy metabolism and intracellular $\mathrm{pH}$ in the ventricles of pig hearts. Methods: Ten isolated pig hearts received 20 minutes of antegrade continuous normothermic blood cardioplegic perfusion for collection of control (baseline) data, followed by 60 minutes of either antegrade continuous normothermic blood cardioplegic perfusion $(n=5)$ or retrograde continuous normothermic blood cardioplegic perfusion ( $=5$ ) with occlusion of the left anterior descending and the right coronary arteries. The hearts were then subjected to antegrade continuous normothermic blood cardioplegic perfusion for 20 minutes. The perfusion pressures were maintained between 80 and $100 \mathrm{~mm} \mathrm{Hg}$ and between 38 and $43 \mathrm{~mm} \mathrm{Hg}$ during antegrade and retrograde continuous normothermic blood cardioplegic perfusions, respectively. Intracellular $\mathrm{pH}$ and creatine phosphate, inorganic phosphate, and adenosine triphosphate levels were measured continuously in each ventricle by means of localized phosphorus 31 magnetic resonance spectroscopy with 2 surface coils. Results: Both antegrade and retrograde continuous normothermic blood cardioplegic perfusion resulted in a significant increase in inorganic phosphate level and decreases in creatine phosphate level, adenosine triphosphate level, and intracellular pH. No significant differences in these changes were observed between the two groups. The creatine phosphate and adenosine triphosphate levels were significantly lower in the right ventricle than in the left ventricle during retrograde continuous normothermic blood cardioplegic perfusion. On reperfusion, the inorganic phosphate level, creatine phosphate level, and intracellular pH recovered completely; however, no recovery in the adenosine triphosphate level was seen in the ventricles of either group. Conclusions: Retrograde continuous normothermic blood cardioplegic perfusion does not provide better protection to ischemic areas of the ventricles than does antegrade continuous normothermic blood cardioplegic perfusion under our experimental conditions. (J Thorac Cardiovasc Surg 1999;117:9941003)
From the Institute for Biodiagnostics, National Research Council, Winnipeg, Manitoba, Canada, ${ }^{\mathrm{a}}$ and the Department of Cardiothoracic Surgery, State University of New York at Buffalo, Buffalo, NY.

Supported by the National Research Council of Canada.

Received for publication April 20, 1998; revisions requested July 7, 1998; revisions received Dec 8, 1998; accepted for publication Jan 11, 1999.

Address for reprints: Roxanne Deslauriers, PhD, or Jian Ye, MD, Institute for Biodiagnostics, National Research Council of Canada, 435 Ellice Ave, Winnipeg, Manitoba R3B 1Y6, Canada. Copyright $(\odot) 1999$ by Mosby, Inc.

$0022-5223 / 99 \$ 8.00+0 \quad \mathbf{1 2 / 1 / 9 7 0 5 2}$
$W$ arm blood cardioplegic perfusion has been proposed as a better method of myocardial protection than conventional hypothermic cardioplegic regimens because of better oxygen delivery, better adenosine triphosphate (ATP) generation, and avoidance of the negative effects of hypothermia. ${ }^{1-4}$ Administration of cardioplegic solutions through the coronary sinus has gained importance in cardiac operations because of the simplicity of the transatrial approach. ${ }^{5}$ Clinical and 
experimental reports have demonstrated good results with retrograde continuous normothermic blood cardioplegic perfusion in patients undergoing heart surgery. ${ }^{1,6,7}$ Research into retrograde continuous normothermic blood cardioplegic perfusion is still limited, however, and results are controversial. ${ }^{8,9}$ Nonuniform delivery of cardioplegic solution to the right ventricle has been noted during retrograde perfusion. ${ }^{10,11}$ Our previous study with isolated normal pig hearts showed that retrograde continuous normothermic blood cardioplegic perfusion does not preserve normal energy metabolism and provides less protection to the right ventricle than to the left ventricle. ${ }^{12}$

Although it has been demonstrated both in animals and in human beings that retrograde cold cardioplegic perfusion provides better protection to an acute ischemic area than does antegrade cold cardioplegic perfusion, it has not been determined whether retrograde continuous normothermic blood cardioplegic perfusion provides better protection to ischemic areas of the left and right ventricles than does antegrade continuous normothermic blood cardioplegic perfusion. In this study localized phosphorus 31 magnetic resonance (MR) spectroscopy with 2 surface coils ${ }^{12}$ was used during antegrade and retrograde continuous normothermic blood cardioplegic perfusion to monitor the changes in energy metabolism and intracellular $\mathrm{pH}$ in ischemic areas of the right and the left ventricles.

\section{Material and methods}

Surgical preparation. Ten pigs (commercial farm Yorkshire cross-bred) of either sex weighing 28 to $35 \mathrm{~kg}$ were used in this study and received humane care according to the "Guide to the Care and Use of Experimental Animals" published by the Canadian Council on Animal Care. ${ }^{13}$ All animals were medicated before the study intramuscularly with ketamine hydrochloride $(20 \mathrm{mg} / \mathrm{kg})$, xylazine $(2.2 \mathrm{mg} / \mathrm{kg})$, and atropine sulfate $(0.03 \mathrm{mg} / \mathrm{kg})$. Anesthesia was initiated with $4 \%$ isoflurane and maintained with 1.5 to $2 \%$ isoflurane through an anesthesia system (Ohmeda Excel 210; DatexOhmeda Division, Instrumentarium Corp, Helsinki, Finland). Approximately $300 \mathrm{~mL}$ blood was withdrawn from the pig after all cannulation was finished, and this was used as a minimal prime for the perfusion system.

As described previously, ${ }^{12,14}$ a median sternotomy was performed and the heart was exposed after the pericardium was opened. Intravenous heparin was administered at $500 \mathrm{U} / \mathrm{kg}$. The ascending aorta was cannulated with an RMI 9F, triplelumen antegrade cardioplegic cannula (Canadian Cardiovascular, Mississauga, Ontario, Canada). The 3 lumina simultaneously provided a pressure line, a perfusion line, and a vent or venous sampling port during retrograde continuous normothermic blood cardioplegic perfusion. After occlusion of the branches of the aortic arch and the aortic arch, perfu- sion down the aortic root was initiated with oxygenated blood by means of a membrane oxygenator (CAPIOX 308; Terumo Corporation, Tokyo, Japan) with a roller pump (COBE model C22.2; COBE BCT, Inc, Lakewood, Colo). At the same time the superior and inferior venae cavae were clamped and the pulmonary artery and both atria were opened to prevent distention of the heart. The perfusion pressure in the aorta was monitored continuously and maintained at 80 to $100 \mathrm{~mm} \mathrm{Hg}$ with a concurrent flow rate of 100 to $200 \mathrm{~mL} / \mathrm{min}$. The heart and lungs were isolated en bloc, with care taken not to cut the esophagus to avoid contamination of the collected blood. The weight of the heart was distributed equally through suspension from the aorta to avoid aortic valve incompetence caused by aortic distortion. The hemiazygos vein was closed with a purse-string suture and the coronary sinus was cannulated with a Sarns $13 \mathrm{~F}$ dual-lumen retrograde cardioplegic perfusion cannula with manual inflatable balloon (3M Health Care, Ann Arbor, Mich), thus providing retroperfusion and pressure monitoring. The cannula was advanced to just before the posterior vein of the left ventricle. Segments about $0.5 \mathrm{~cm}$ long of both the left anterior descending coronary artery and the right coronary artery were isolated and a hydrovascular occluder (In Vivo Metric, Healdsburg, Calif) was placed around each artery. Heart arrest was achieved by adding potassium chloride to the reservoir to reach a final concentration of $18 \pm 2 \mathrm{mmol} / \mathrm{L}$. The hematocrit value was maintained at $17 \%$ to $22 \%$ (the reference value for pigs is approximately $30 \%$ ). The $\mathrm{PCO}_{2}$ and $\mathrm{Po}_{2}$ were maintained between 35 and 45 $\mathrm{mm} \mathrm{Hg}$ and between 300 and $440 \mathrm{~mm} \mathrm{Hg}$, respectively, by adjusting the total gas flow and the percentage of carbon dioxide gas. Blood $\mathrm{pH}$ was maintained between 7.35 and 7.45. All the electrolyte levels except that of potassium were kept within physiologic ranges. The temperature was maintained at $37^{\circ} \mathrm{C}$ with a circulated warm water bath system (Lauda Dr R. Wobser Gmbh \& Co KG, Postfach, Germany).

The perfusion system was designed to allow remote switching to either antegrade or retrograde continuous normothermic blood cardioplegic perfusion from outside the MR instrument to avoid any repositioning of the heart probe. During antegrade continuous normothermic blood cardioplegic perfusion the perfusion pressure measured at the ascending aorta was maintained at 80 to $100 \mathrm{~mm} \mathrm{Hg}$ with a concurrent pump flow of 100 to $200 \mathrm{~mL} / \mathrm{min}$ before and after occlusion of the coronary arteries and of 80 to $100 \mathrm{~mL} / \mathrm{min}$ during occlusion of the coronary arteries. A retrograde perfusion pressure of 38 to $43 \mathrm{~mm} \mathrm{Hg}$ at the coronary sinus was achieved by adjusting the pump flow (120-200 $\mathrm{mL} / \mathrm{min})$.

Protocol. The protocol for this study was 20 minutes of antegrade continuous normothermic blood cardioplegic perfusion (baseline), then 60 minutes of either antegrade or retrograde continuous normothermic blood cardioplegic perfusion with occlusion of the left anterior descending and right coronary arteries, and then finally 20 minutes of reperfusion with antegrade continuous normothermic blood cardioplegic perfusion. Ten pigs were randomly divided into 2 groups, the antegrade group $(n=5)$ and the retrograde group $(n=5)$. 
MR spectroscopy. As described previously, ${ }^{12}$ all experiments were performed with a Bruker MSLX spectrometer (Bruker Instruments Inc, Billerica, Mass) equipped with a 7$\mathrm{T}$, 40-cm horizontal-bore magnet. Two similar surface coils $15 \mathrm{~mm}$ in diameter were used; one was placed against the left ventricular wall (over the area supplied by the left anterior descending coronary artery) and the other was placed against the right ventricular wall (over the area supplied by the right coronary artery). The separation of the 2 coils was about 10 $\mathrm{cm}$. The acquisition of data from the 2 coils was time-interleaved; only 1 coil was connected to the spectrometer at a time. Spectra were obtained alternately every 2 minutes from the left and right ventricles by means of a single pulse-andacquire sequence. The pulse length was $60 \mu \mathrm{s}$, and 1024 complex data points were acquired in $102 \mathrm{~ms}$ after the excitation pulse. The repetition time was 2 seconds, and 60 acquisitions were averaged in 2 minutes. This repetition time was used for signal-to-noise considerations. Although the metabolites are not fully relaxed with a repetition time of 2 seconds, the percentage change in each metabolite is not affected by partial saturation in the absence of any change in relaxation times of the metabolites. Field homogeneity was optimized by global shimming on the proton signal. ${ }^{12}$

Release of creatine kinase. Afferent and efferent blood samples were obtained before, during, and after occlusion of the left anterior descending and right coronary arteries for creatine kinase (CK) measurements. Activity of CK was determined by an enzymatic reaction method (Sigma CK-10, Sigma Chemical Co, St Louis, Mo) monitored at $340 \mathrm{~nm}$ (DU 650 spectrophotometer, Beckman Coulter, Inc, Fullerton, Calif), according to the procedures described in the manufacturer's manual. CK release was calculated according to the following formula: CK release (in units per minute per 100 grams of tissue $)=($ CK level in efferent blood [in units per liter] - CK level in afferent blood [in units per liter]) $\times$ Antegrade or retrograde perfusion flow (in liters per minute) $\div$ Heart weight (in grams)

Needle biopsies for high-performance liquid chromatographic analyses. Transmural biopsy specimens were obtained with a biopsy needle from the nonischemic and ischemic areas of the left and right ventricles just before completion of the experiments and were immediately immersed in liquid nitrogen. The tissue samples were then freeze-dried (The VirTis Company, Gardiner, NY) to determine myocardial concentrations of ATP, adenosine diphosphate, adenosine monophosphate, creatine phosphate, and creatine. The compounds were measured by high-performance liquid chromatography (HPLC; Waters 510 pump, Waters 996 Photodiode Array Detector; Waters Corporation, Chromatography Division, Milford, Mass) with the gradient pairedion chromatographic technique described previously. ${ }^{15}$

Tissue water content. Tissue samples of approximately 2 $\mathrm{g}$ obtained at the end of the experiments from the nonischemic and ischemic areas of the left and right ventricles were incubated at $60^{\circ} \mathrm{C}$ until constant weight was reached (approximately 4 days). Wet and dried tissue weights were obtained before and after incubation, respectively. Tissue water content (as a percentage) was calculated according to the following formula: Tissue water content $(\%)=($ Wet tissue weight - Dried tissue weight)/Wet tissue weight $\times 100 \%$.

Data analysis. The heights of the MR spectral peaks were used to determine the relative changes in inorganic phosphate, creatine phosphate, and ATP, because the width of the MR signals did not change during the course of the experiments. The heights of MR spectral peaks were obtained by means of inhouse analysis software (Allfit). ${ }^{16}$ The intracellular $\mathrm{pH}$ was calculated from the chemical shift of the inorganic phosphate peak. ${ }^{17}$ Three peaks arising from ATP were observed in the MR spectra $(\gamma, \alpha$, and $\beta$ ). The $\beta$-ATP peak was used to follow the changes in ATP. The values of the $\beta$-peak of ATP and the peaks of creatine phosphate, inorganic phosphate and intracellular $\mathrm{pH}$ during the initial control antegrade continuous normothermic blood cardioplegic perfusion period were averaged across 4 spectra and used as baseline levels. During occlusion of the coronary arteries and reperfusion the values of the $\beta$-peak of ATP and the peaks of creatine phosphate, inorganic phosphate, and intracellular $\mathrm{pH}$ were averages of 2 spectra and expressed as a percentage of the baseline level $(100 \%)$ obtained during initial antegrade cardioplegic perfusion before occlusion of the coronary arteries.

The data are presented as the mean \pm SEM. A repeatedmeasures analysis of variance and the Duncan test were used for comparison of HPLC data from different regions within the group and for comparison of MR spectroscopic data at different time points within the group. Simultaneous $95 \%$ confidence intervals were calculated with the Bonferroni method. ${ }^{18}$ Student $t$ tests were performed to compare the data (MR spectroscopy, HPLC, tissue water content, and CK release) between the antegrade and retrograde groups or between the left and right ventricles. In addition, standard 95\% confidence intervals were calculated for comparison between the antegrade and retrograde groups. The statistical software used for data analysis was STATISTICA 5.1 for Windows (StatSoft Inc, Tulsa, Okla).

\section{Results}

Antegrade versus retrograde continuous normothermic blood cardioplegic perfusion. During initial antegrade continuous normothermic blood cardioplegic perfusion (before occlusion of the coronary arteries), MR spectroscopy showed stable levels of creatine phosphate and ATP, with little evidence of inorganic phosphate and with intracellular $\mathrm{pH}$ within the reference range in both ventricles (left ventricle $7.40 \pm$ 0.05 and right ventricle $7.36 \pm 0.05$ ) in both groups. After occlusion of the coronary arteries neither antegrade nor retrograde continuous normothermic blood cardioplegic perfusion maintained normal aerobic energy metabolism in the ischemic areas of the ventricles, as indicated by a significant increase in the inorganic phosphate level (Fig 1) and decreases in the creatine phosphate level (Fig 2), ATP level (Fig 3), and 


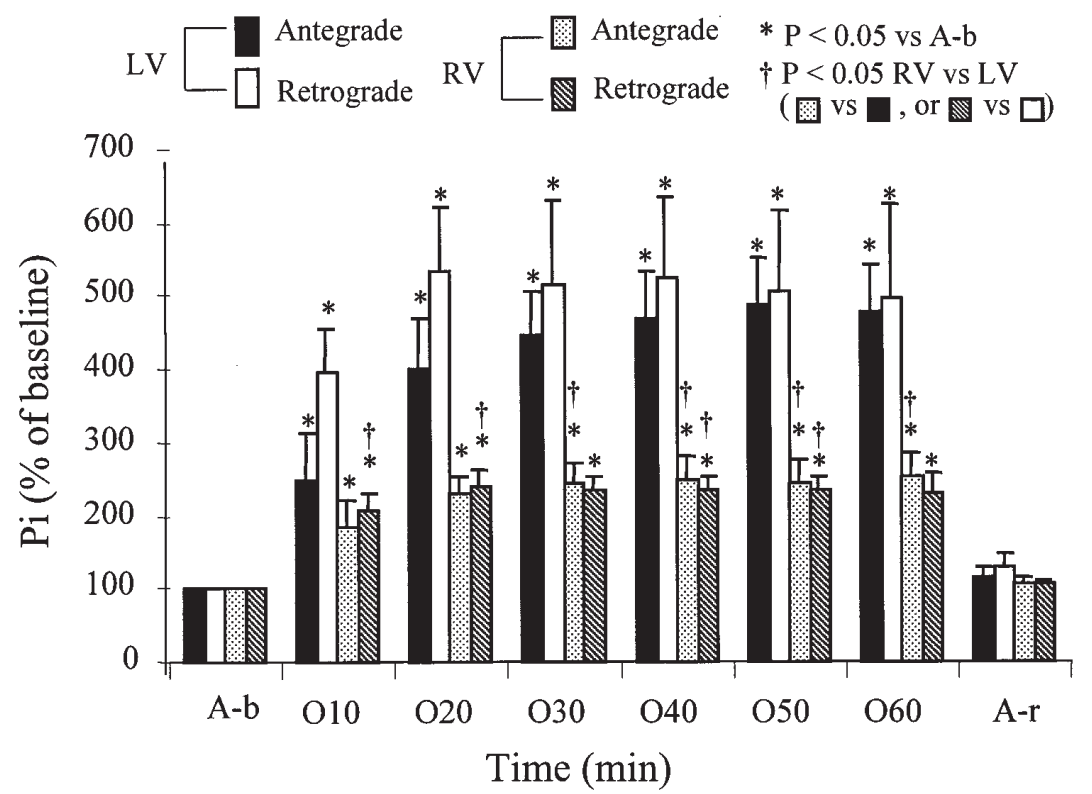

Fig 1. Inorganic phosphate $(P i)$ level in ischemic area of left ventricle $(L V)$ and right ventricle $(R V)$ during antegrade and retrograde cardioplegic perfusion. Level of inorganic phosphate during initial antegrade cardioplegic perfusion $(A-b)$ was used as baseline $(100 \%)$. $O$, Occlusion of coronary arteries; $A-r$, antegrade cardioplegic perfusion reperfusion. Error bars represent SEM.

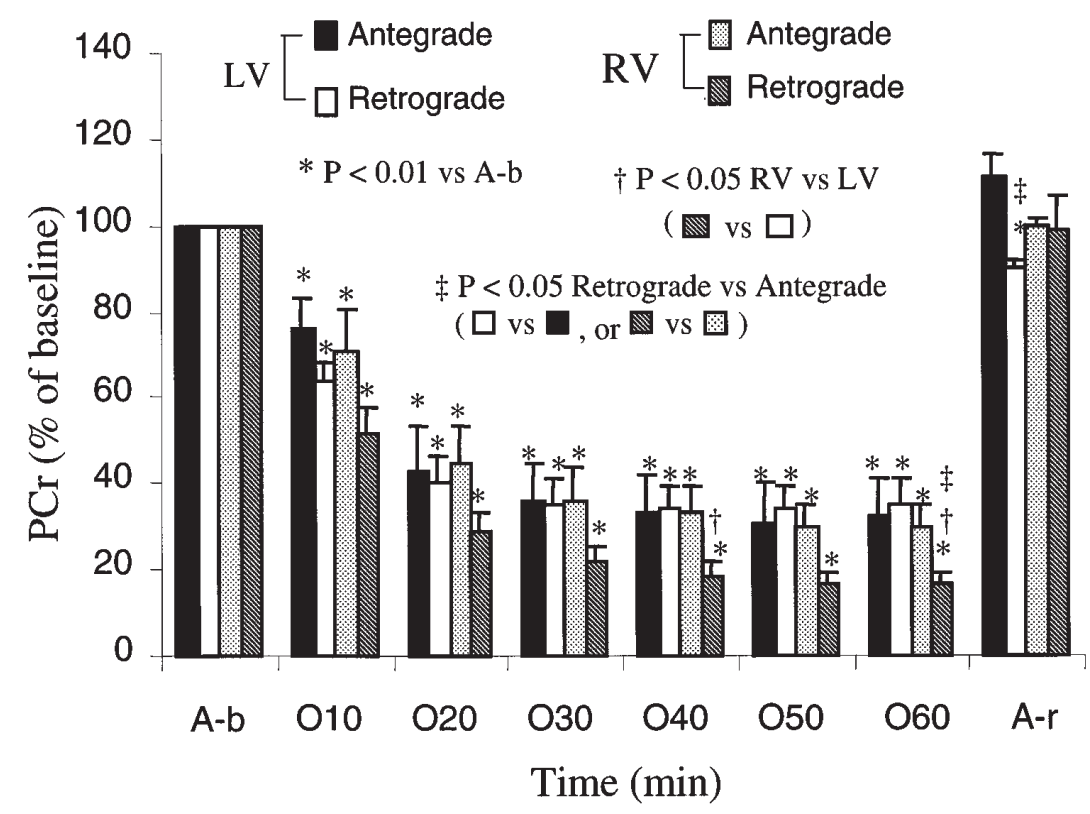

Fig 2. Creatine phosphate $(P C r)$ level in ischemic area of left ventricle $(L V)$ and right ventricle $(R V)$ during antegrade and retrograde cardioplegic perfusion. Level of creatine phosphate during initial antegrade cardioplegic perfusion $(A-b)$ was used as baseline (100\%). $O$, Occlusion of coronary arteries; $A$ - $r$, antegrade cardioplegic perfusion reperfusion. Error bars represent SEM.

intracellular $\mathrm{pH}$ (Fig 4). Among these changes, the increase in the inorganic phosphate level and the decrease in the level of creatine phosphate reached maxima at 20 to 30 minutes of occlusion with both antegrade and retrograde continuous normothermic blood cardioplegic perfusion. No significant difference 


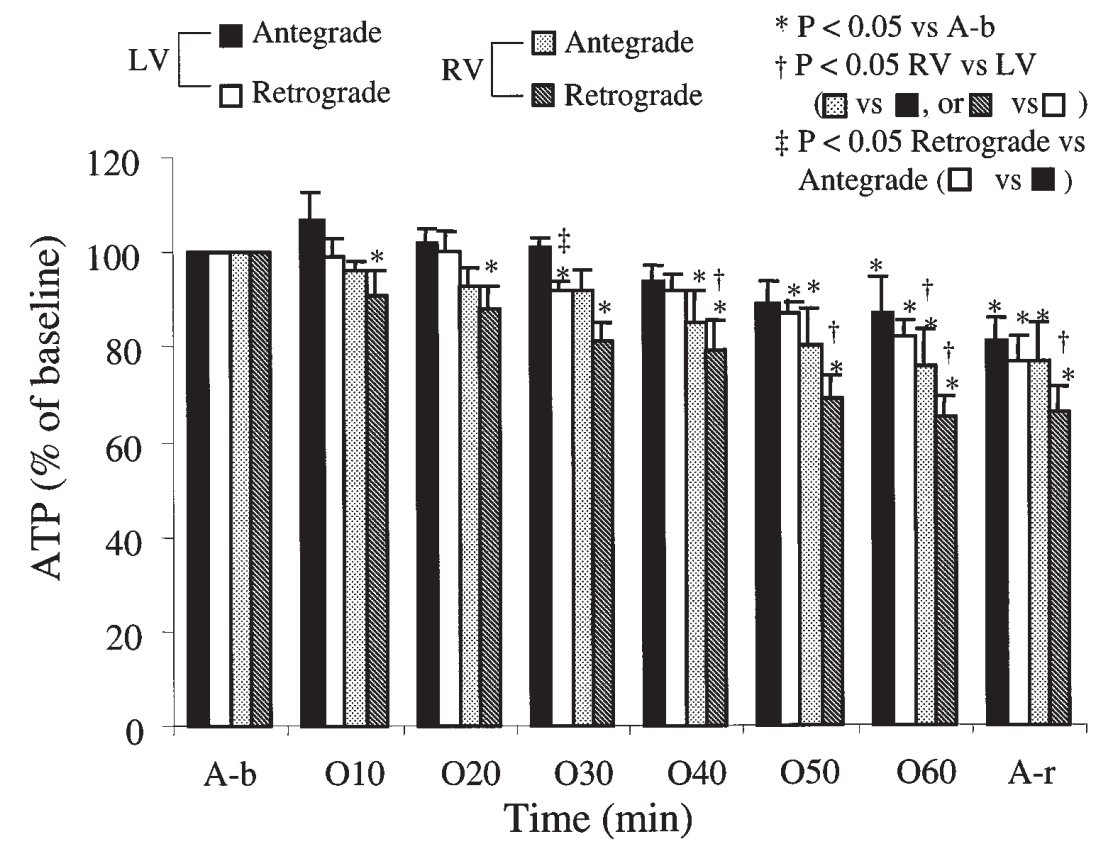

Fig 3. ATP level in ischemic area of left ventricle $(L V)$ and right ventricle $(R V)$ during antegrade and retrograde cardioplegic perfusion. Level of ATP during initial antegrade cardioplegic perfusion $(A-b)$ was used as baseline $(100 \%)$. $O$, Occlusion of coronary arteries; $A-r$, antegrade cardioplegic perfusion reperfusion. Error bars represent SEM.

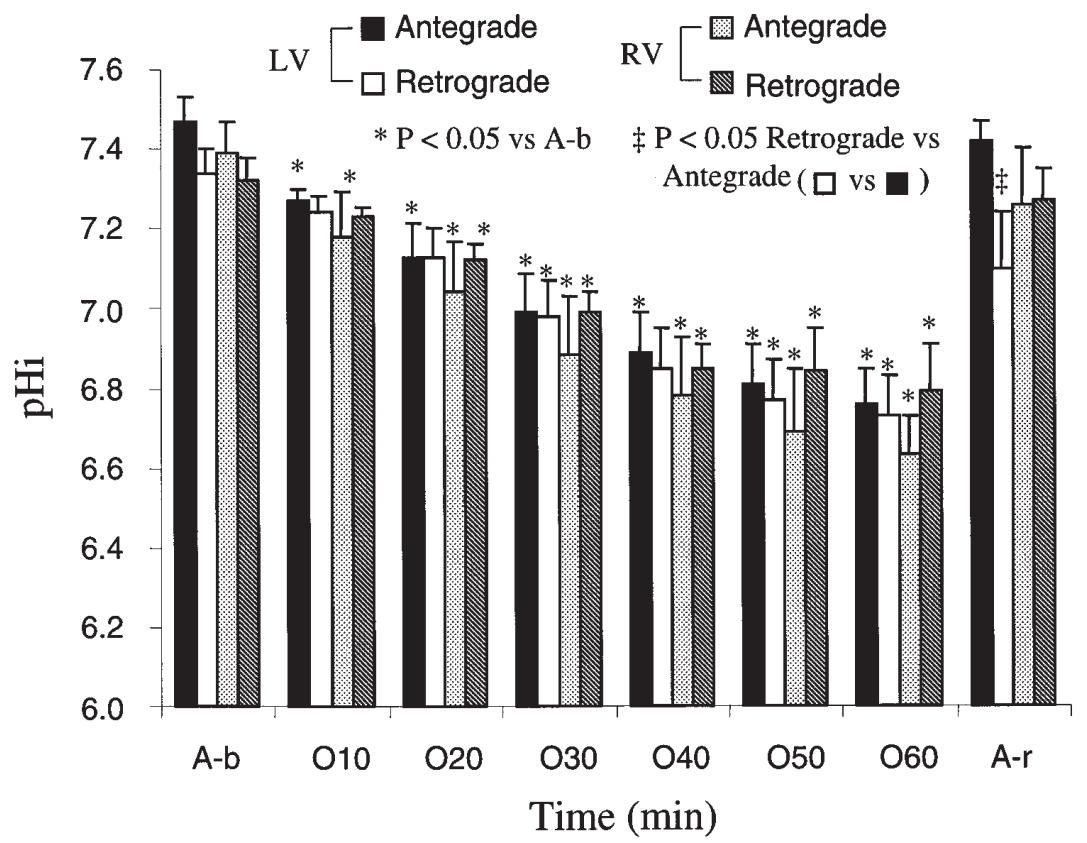

Fig 4. Intracellular $\mathrm{pH}$ in ischemic area of left ventricle $(L V)$ and right ventricle $(R V)$ during antegrade and retrograde cardioplegic perfusion. $A-b$, antegrade cardioplegic perfusion baseline; $O$, occlusion of coronary arteries; $A-r$, antegrade cardioplegic perfusion reperfusion. Error bars represent SEM.

was observed between the antegrade and retrograde groups (Figs 1 and 2). A continuous decrease in the ATP level was observed in the ischemic areas in both groups during the 60-minute occlusion of the coronary arteries. No significant difference was seen between the 2 groups (Fig 3). Both antegrade and retrograde 
Table I. Metabolite levels in the left and right ventricles at the end of the experiments

\begin{tabular}{|c|c|c|c|c|c|c|}
\hline \multirow[b]{2}{*}{ Area } & \multicolumn{2}{|c|}{ ATP } & \multicolumn{2}{|c|}{ Creatine phosphate } & \multicolumn{2}{|c|}{ Creatine phosphate + creatine } \\
\hline & Antegrade & Retrograde* $^{*}$ & Antegrade & Retrograde* $^{*}$ & Antegrade & Retrograde* $^{*}$ \\
\hline Left ventricular nonischemic area & $29 \pm 1.0$ & $28 \pm 1.1$ & $66 \pm 7.3$ & $78 \pm 3.1$ & $97 \pm 4.4$ & $104 \pm 4.5$ \\
\hline Left ventricular ischemic area & $24 \pm 2.7$ & $23 \pm 2.3$ & $78 \pm 5.0$ & $76 \pm 8.1$ & $100 \pm 6.5$ & $102 \pm 8.5$ \\
\hline Statistical significance & $P=.025 \dagger$ & $P=.021 \dagger$ & & & & \\
\hline $\begin{array}{l}\text { Right ventricular nonischemic area } \\
\text { Statistical significance }\end{array}$ & $26 \pm 1.5$ & $\begin{aligned} 21 & \pm 2.5 \\
P & =.017\end{aligned}$ & $57 \pm 3.3$ & $60 \pm 8.1$ & $\begin{aligned} 79 & \pm 3.2 \\
P & =.004 \ddagger\end{aligned}$ & $\begin{aligned} 80 & \pm 7.1 \ddagger \\
P & =.014 \ddagger\end{aligned}$ \\
\hline $\begin{array}{l}\text { Right ventricular ischemic area } \\
\text { Statistical significance }\end{array}$ & $\begin{aligned} 21 & \pm 3.0 \\
P & =.012 \dagger\end{aligned}$ & $18 \pm 1.8$ & $\begin{array}{l}64 \pm 13.2 \\
P=.005\end{array}$ & $76 \pm 3.5$ & $\begin{aligned} 85 & \pm 12.1 \ddagger \\
P & =.002 \ddagger\end{aligned}$ & $93 \pm 2.2$ \\
\hline
\end{tabular}

Data were obtained by HPLC. The concentrations are shown as micromoles per gram dry weight and mean \pm SEM.

*Retrograde continuous normothermic blood cardioplegic perfusion versus antegrade continuous normothermic blood cardioplegic perfusion (no significant difference in any area).

$\dagger P<.05$ (right ventricular ischemic area versus right ventricular nonischemic area or left ventricular ischemic area versus left ventricular nonischemic area within the same group).

$\ddagger P<.05$ (right ventricular nonischemic area versus left ventricular nonischemic area or right ventricular ischemic area versus left ventricular ischemic area within the same group).

Table II. ATP levels in the ischemic areas during reperfusion

\begin{tabular}{lcccc}
\hline & \multicolumn{2}{c}{ Left ventricular ischemic area } & & \multicolumn{2}{c}{ Right ventricular ischemic area } \\
\cline { 2 - 4 } Assay method & Antegrade & Retrograde & Antegrade & Retrograde \\
\hline MR spectroscopy $(\mathrm{n}=5)$ & $81 \pm 4.9$ & $77 \pm 5.3$ & $77 \pm 8.0$ & $66 \pm 5.3$ \\
HPLC $(\mathrm{n}=5) *$ & $82 \pm 2.9$ & $79 \pm 2.5$ & $80 \pm 2.5$ & $70 \pm 2.3$ \\
Statistical significance $\dagger$ & $P=.50$ & $P=.75$ & $P=.74$ & $P=.51$ \\
\hline
\end{tabular}

Values are expressed as percentages relative to reference control levels. The reference control levels for MR spectroscopy were the baseline percentage levels (100\%) obtained during the antegrade cardioplegic perfusion period before occlusion of the coronary arteries. For HPLC the level measured in the nonischemic area of the left ventricle from the antegrade group was used as the reference control value for the ischemic areas of the left ventricle in both antegrade and retrograde groups. The level measured in the nonischemic area of the right ventricle in the antegrade continuous normothermic blood cardioplegic perfusion group was used as the reference control level for the ischemic areas of the right ventricle in both the antegrade and retrograde groups.

*The percentage changes were calculated from the means of 5 experiments in each subgroup (e.g. ischemic area of left ventricle in the antegrade group, ischemic area of left ventricle in the retrograde group, ischemic area of right ventricle in the antegrade group, and ischemic area of right ventricle in the retrograde group) and the $\mathrm{SE}$ of the quotient estimated as root mean square error.

$\dagger$ Student $t$ test.

continuous normothermic blood cardioplegic perfusion resulted in a continuous decrease in intracellular $\mathrm{pH}$ in the ischemic areas of both ventricles during the 60minute occlusion of the coronary arteries (Fig 4). On release of the occlusion and reperfusion with antegrade continuous normothermic blood cardioplegic solution, immediate and complete recoveries of the inorganic phosphate level, creatine phosphate level, and intracellular $\mathrm{pH}$ were observed in both groups; however, the concentrations of ATP did not return to preocclusion values (Figs 1-4).

The levels of metabolites determined by HPLC at the end of the experiments are shown in Table I. The concentration of ATP in the nonischemic myocardium obtained from the antegrade group was similar to our previous finding in normal pig hearts and was used as the reference control value. In both groups the concentration of ATP was significantly lower than the reference control value in the ischemic, reperfused myocardium, indicating incomplete recovery of ATP after reperfusion, which is consistent with the MR spectroscopic findings. No significant difference in ATP concentration was observed between the antegrade and retrograde groups. The level of ATP determined by HPLC was similar to that obtained from MR spectroscopy during reperfusion (Table II). The concentrations of creatine phosphate, creatine, adenosine diphosphate, total creatine (creatine phosphate plus creatine), and adenosine in the ischemic areas were similar to those in the nonischemic areas in the antegrade and retrograde groups. The concentration of adenosine monophosphate was low $(<0.001 \mu \mathrm{mol} / \mathrm{g}$ dry weight $)$ in most specimens.

The release of CK appeared to be higher in the retrograde group than in the antegrade group at 10 minutes of occlusion and 20 minutes of reperfusion; however, no significant difference in CK release was found 


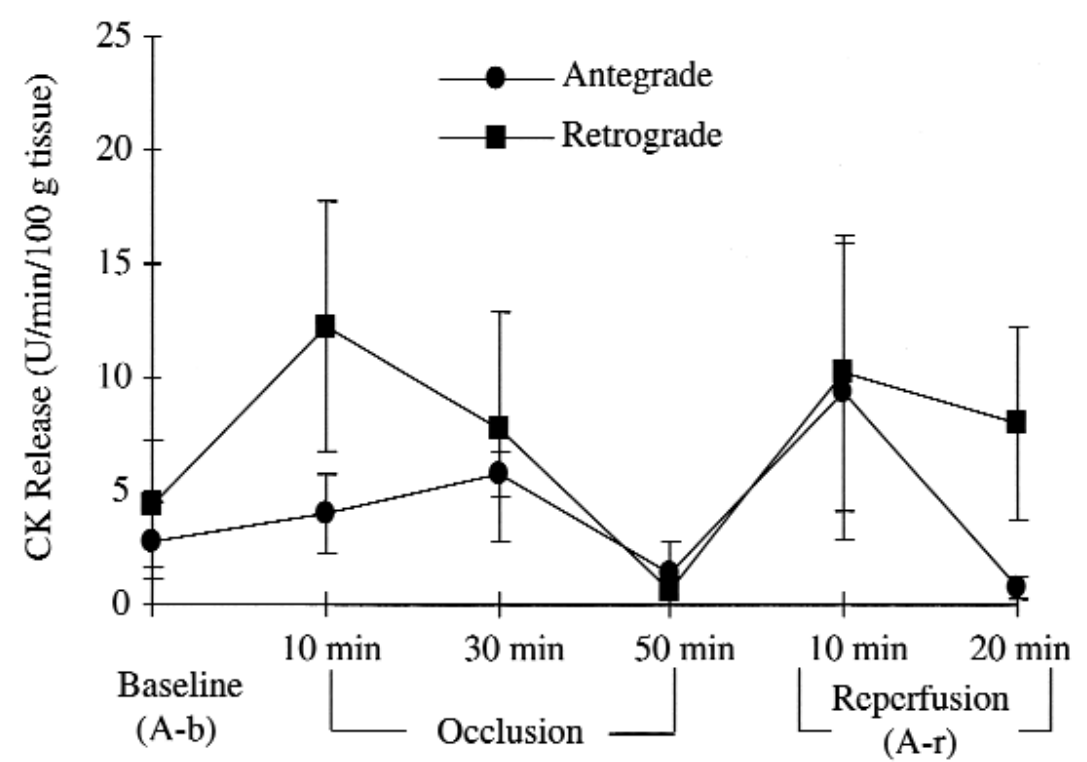

Fig 5. CK release from hearts during antegrade and retrograde cardioplegic perfusion. $A-b$, antegrade cardioplegic perfusion baseline; $A$-r , antegrade cardioplegic perfusion reperfusion. Error bars represent SEM.

between the 2 groups (Fig 5). The tissue water content in the ischemic, reperfused myocardium (left ventricle in antegrade group $85.6 \% \pm 0.5 \%$, left ventricle in retrograde group $86.0 \% \pm 0.9 \%$; right ventricle in antegrade group $86.2 \% \pm 0.7 \%$, right ventricle in retrograde group $86.4 \% \pm 0.2 \%$ ) was similar to that in normal myocardium (left ventricle in antegrade group $86.2 \% \pm$ $0.5 \%$, left ventricle in retrograde group $86.8 \% \pm 0.3 \%$; right ventricle in antegrade group $87.1 \% \pm 0.5 \%$, right ventricle in retrograde group $87.5 \% \pm 0.3 \%$ ), and no difference was observed between the antegrade and retrograde groups.

Right ventricle versus left ventricle. The patterns of changes in the ATP, creatine phosphate, and inorganic phosphate levels and in intracellular $\mathrm{pH}$ during the experiments were similar in the left and right ventricles of both groups (Figs 1-4). However, in both groups more inorganic phosphate accumulated in the left ventricle than in the right ventricle during occlusion of the coronary arteries (Fig 1). The reductions in creatine phosphate and ATP concentrations seemed to be more severe and rapid during occlusion of the coronary arteries in the retrograde group in the right ventricle than in the left ventricle (Figs 2 and 3). The intracellular $\mathrm{pH}$ showed similar decreases during occlusion of the coronary arteries in both ventricles in both groups (Fig 4). Inorganic phosphate level, creatine phosphate level, and intracellular $\mathrm{pH}$, but not ATP level, returned to baseline after release of the occlusion and reperfusion in both ventricles of both groups.
No difference in tissue water content was observed between the 2 ventricles in either group. According to HPLC measurements, during retrograde continuous normothermic blood cardioplegic perfusion the concentrations of ATP in the normal and ischemic myocardium appeared to be lower in the right ventricle than in the left ventricle (Table I). This indicates that some injury occurred not only in the ischemic zone but also in the nonischemic zone of the right ventricle during retrograde continuous normothermic blood cardioplegic perfusion, which is consistent with our previous finding in normal pig hearts. ${ }^{12}$ Significantly lower concentrations of total creatine (creatine phosphate plus creatine) were observed in both the ischemic and nonischemic areas of the right ventricle than in the respective areas of the left ventricle in both the retrograde and antegrade groups (Table I).

\section{Discussion}

Under our experimental conditions the significant findings were as follows: (1) Neither antegrade nor retrograde continuous normothermic blood cardioplegic perfusion completely prevented ischemic energy metabolism in the ischemic area. Retrograde continuous normothermic blood cardioplegic perfusion did not provide better protection to the ischemic area than did antegrade continuous normothermic blood cardioplegic perfusion. These findings are different from those obtained with cold cardioplegic perfusion. (2) The ischemic area of the right ventricle appeared to be less protected than that 
of the left ventricle during retrograde continuous normothermic blood cardioplegic perfusion. (3) Damage to the ischemic area did not appear to be severe during 60 minutes of either antegrade or retrograde continuous normothermic blood cardioplegic perfusion, as indicated by complete recovery of creatine phosphate and inorganic phosphate levels and of intracellular $\mathrm{pH}$, by the lack of a significant release of $\mathrm{CK}$, and by the lack of an increase in tissue water in the ischemic area after reperfusion. The small reduction in ATP concentration observed in this study does not appear to be sufficiently severe to cause irreversible damage to myocardium. It has been reported that a decrease in ATP content to less than $35 \%$ to $40 \%$ of preischemic levels is associated with irreversible damage. ${ }^{19,20}$

The efferent blood samples used to determine the release of $\mathrm{CK}$ were obtained from the ascending aorta during retrograde continuous normothermic blood cardioplegic perfusion. The retrograde blood flow that passed through the myocardium and returned to the aorta was much lower than the total retrograde perfusion flow because of a large amount of shunting of blood flow to the ventricular chambers. The release of CK during retrograde continuous normothermic blood cardioplegic perfusion may be overestimated because total retrograde perfusion flow, rather than retrograde flow through the myocardial tissue, was used to calculate the release of CK.

Retrograde continuous normothermic blood cardioplegic perfusion has been enthusiastically endorsed by several investigators, and its use has become widespread. Blood distribution during retrograde flow of normothermic blood cardioplegic perfusion to the heart, however, is controversial. Gundry and coworkers $^{21}$ found that regional myocardial perfusion, including the right side of the heart, is uniform during warm continuous retrograde cardioplegic perfusion in human beings. Other investigators have demonstrated that perfusion deficits exist during retrograde warm blood cardioplegic perfusion, especially in the right ventricle. ${ }^{10}$ Our previous study also demonstrated that retrograde continuous normothermic blood cardioplegic perfusion does not supply sufficient oxygen to maintain normal energy metabolism in both ventricles, especially the right ventricle, of normal hearts. ${ }^{12}$ In this study we sought to assess the metabolic effects of antegrade and retrograde continuous normothermic blood cardioplegic perfusion on the ischemic areas of each ventricle in swine hearts with acute and complete occlusion of 2 major coronary arteries and to investigate whether retrograde continuous normothermic blood cardioplegic perfusion provides better protection to the ischemic area than does antegrade continuous normothermic blood cardioplegic perfusion. The absence of ischemic metabolism would provide evidence for adequate oxygen supply to the tissue. We have shown that neither antegrade nor retrograde continuous normothermic blood cardioplegic perfusion, as applied under our conditions in a pig heart, maintains myocardial aerobic metabolism in the ischemic areas of the right and left ventricles, as indicated by significant declines in creatine phosphate and ATP levels and in intracellular $\mathrm{pH}$, as well as by the increase in the inorganic phosphate levels. Retrograde continuous normothermic blood cardioplegic perfusion did not provide better preservation of normal energy metabolism in the ischemic area than antegrade continuous normothermic blood cardioplegic perfusion. Retrograde continuous normothermic blood cardioplegic perfusion also appeared to result in more severe ischemic metabolism in the right ventricle.

The findings indicate that retrograde continuous normothermic blood cardioplegic perfusion does not provide sufficient oxygen to the ischemic myocardium to maintain normal metabolism at normal temperature. These findings can be explained as follows: First, poor capillary flow (nutritive flow) has been found in even normal hearts as a result of shunting of flow away from the capillary beds. A large amount of cardioplegic solution administered retrogradely travels through venous collaterals, including the Thebesian veins, and drains directly into the right ventricle. ${ }^{11,22}$ In studies with a microsphere technique, it has been reported that the nonnutritive flow of retrograde perfusion is between $64 \%$ and $74 \%,{ }^{10,23,24}$ which may underestimate true nonnutritive flow as determined by capillary-dependent radioisotope uptake. ${ }^{25}$ The limited nutritive flow supplied by retrograde perfusion is inadequate to maintain aerobic metabolism in arrested normothermic hearts. ${ }^{12}$ Second, the model used in the study involves acute occlusion of coronary arteries, and therefore only previously formed collateral vessels exist in this model; these would be fewer than those found in hearts with chronically occluded coronary arteries. It is believed that less nutritive flow can reach the ischemic area in this acute ischemic model than in chronically ischemic hearts. Third, although oxygen consumption is reduced in arrested hearts, it remains greater under the conditions of normothermia than those of hypothermia. Therefore the limited amount of oxygen provided by retrograde perfusion may be sufficient to maintain normal metabolism during hypothermia (cold cardioplegic perfusion) but is insufficient under conditions of normothermia (normothermic cardioplegic perfusion). If only retrograde cardioplegic perfusion is used in severely ischemic hearts, hypothermia may be necessary to further reduce oxygen demands in accordance with the limited oxygen supply. Under these circum- 
stances retrograde cold cardioplegic perfusion may provide better protection to the ischemic zone than retrograde warm cardioplegic perfusion. Because our experiments were not designed to address differences between cold and warm cardioplegic perfusion, however, we are unable to provide any further conclusions on this issue, particularly in this isolated model with acute complete occlusion of coronary arteries. Fourth, to simulate a clinical situation a retrograde catheter with an inflatable balloon was used and placed in the coronary sinus for retrograde delivery of cardioplegic solution. The inflatable balloon may have blocked the middle and small cardiac veins because these 2 veins open to the proximal part of the coronary sinus, as in human hearts. Less retrograde flow could therefore be delivered to the ventricles. This problem may be resolved by placing purse-string sutures around the orifice of the coronary sinus, rather than using an inflatable balloon. ${ }^{26}$ However, the suturing technique is not clinically practical. Finally, in this model of complete occlusion of 2 major coronary arteries, high resistance occurs in the arterial system of the ischemic area. This may result in more shunting of retrograde flow into nonischemic areas or into the ventricular chambers. A recent study has shown that retrograde cardioplegic perfusion does not improve the distribution of cardioplegic solution in either the acutely ischemic myocardial area or the zone of acute infarction in the dog. ${ }^{27}$

One study has shown that retrograde warm blood cardioplegic perfusion preserves myocardial function in the heart with an acute coronary arterial obstruction better than does antegrade warm blood cardioplegic perfusion. ${ }^{6}$ On the basis of the changes in energy metabolites observed in our study, however, retrograde warm blood cardioplegic perfusion does not appear to preserve energy metabolism in the ischemic area better than does antegrade warm blood cardioplegic perfusion. It has also been reported that retrograde warm blood cardioplegic perfusion results in greater anaerobic lactate production during and after cardioplegic arrest than do other cardioplegic techniques. ${ }^{28,29}$

Our study also shows that during retrograde continuous normothermic blood cardioplegic perfusion the ischemic area of the right ventricle is less well protected than that of the left ventricle, as indicated by more severe reductions in creatine phosphate and ATP levels in the right ventricle during 60 minutes of occlusion of the coronary arteries. This may be the result of less nutritive flow to the right ventricle than to the left ventricle as a result of large shunting ${ }^{10,11}$ and possible blockage of the middle and small cardiac veins by the retrograde catheter (inflated balloon) during retrograde cardioplegic perfusion. In this study HPLC data also showed that ATP was significantly reduced in even the nonischemic area of the right ventricle during retrograde continuous normothermic blood cardioplegic perfusion, which is similar to our previous findings in normal pig hearts. ${ }^{12}$ The failure of the ATP level to recover indicates that retrograde continuous normothermic blood cardioplegic perfusion results in some damage to both the normal area and the area of coronary occlusion. The concentration of total creatine (creatine phosphate plus creatine) was lower in the right ventricle than in the left ventricle in both the antegrade and the retrograde groups. This may not indicate any loss of creatine, because the concentration of total creatine in the normal area is also lower in the right ventricle than in the left ventricle ( $81 \%$ of the left ventricle value; Table I).

This study is limited by the lack of functional assessment of the heart after normokalemic reperfusion. Such studies were not performed because of the considerable technical difficulty of performing separate MR measurements on 2 beating ventricles. In addition, this study was performed in acutely ischemic isolated hearts. The effects of both noncoronary collateral flow in the in vivo situation and newly formed collateral circulations in the diseased heart with chronically occluded coronary arteries were absent. The data obtained with this model thus cannot be completely translated into the clinical situation. However, animal models provide controlled experimental conditions and allow measurements to be performed that are often not feasible in human beings.

These results demonstrate that under our experimental conditions retrograde continuous normothermic blood cardioplegic perfusion does not provide better protection of high-energy metabolites and intracellular $\mathrm{pH}$ in the ischemic area of hearts with acute and complete occlusion of coronary arteries than does antegrade continuous normothermic blood cardioplegic perfusion. During retrograde continuous normothermic blood cardioplegic perfusion an ischemic area in the right ventricle appears to be less protected than an ischemic area in the left ventricle.

We thank Lori Shoemaker for biochemical analyses (HPLC) and Monique St-Jean, Rachelle Perchaluk, and Shelly Germscheid for technical assistance.

\section{REFERENCES}

1. Salerno TA, Houck JP, Barrozo CA, Panos A, Christakis GT, Abel JG, et al. Retrograde continuous warm blood cardioplegia: a new concept in myocardial protection. Ann Thorac Surg 1991; 51:245-7.

2. Ali IS, AL-Nowaiser O, Deslauriers R, Panos AL, Barrozo CAM, 
Khan SJ, et al. Continuous normothermic blood cardioplegia. Semin Thorac Cardiovasc Surg 1993;5:141-50.

3. Kurihara S, Sakai T. Effects of rapid cooling on mechanical and electrical responses in ventricular muscle of guinea pig. J Physiol (Lond) 1985;361:361-78.

4. Kaijser L, Jansson E, Schmidt W, Bonfin V. Myocardial energy depletion during hypothermic cardioplegia for cardiac operations. J Thorac Cardiovasc Surg 1985;90:896-900.

5. Gundry SR, Sequiera A, Razzouk AM, McLaughlin JS, Bailey LL. Facile retrograde cardioplegia: transatrial cannulation of the coronary sinus. Ann Thorac Surg 1990;50:882-7.

6. Misare BD, Krukenkamp IB, Lazer ZP, Levitsky S. Retrograde is superior to antegrade continuous warm blood cardioplegia for acute cardiac ischemia. Circulation 1992;86(Suppl):II393-7.

7. Yau TM, Weisel RD, Mickle DA, Ivanow J, Mohabeer MK, Tumisti L, et al. Optimal delivery of blood cardioplegia. Circulation 1991;84(Suppl):III380-8.

8. Allen BS, Winkelmann JW, Hanafy H, Hartz RS, Bolling KS, Ham J, et al. Retrograde cardioplegia does not adequately perfuse the right ventricle. J Thorac Cardiovasc Surg 1995;109:1116-24.

9. Gundry SR, Wang N, Bannon D, Vigesaa RE, Eke C, Pain S, et al. Retrograde continuous warm blood cardioplegia: maintenance of myocardial homeostasis in humans. Ann Thorac Surg 1993; 55:358-61.

10. Caldarone CA, Krukenkamp IB, Misare BD, Levitsky S. Perfusion deficits with retrograde warm blood cardioplegia. Ann Thorac Surg 1994;57:403-6.

11. Smith MV, Stahl RF, Cronin C, Hsi C, Li JM, BelleIsle J, et al. Retrograde coronary sinus perfusion provides nonhomogeneous myocardial blood flow. Cardiovasc Surg 1993;83:331-8.

12. Ye J, Sun J, Shen J, Gregorash L, Summers R, Salerno TA, et al. Does retrograde warm blood cardioplegia provide equal protection to both ventricles? Circulation 1997;96(Suppl):II210-5.

13. Olfert ED, Cross BM, McWilliam AA, editors. Guide to the care and use of experimental animals. Canadian Council on Animal Care. 2nd ed. Ottawa: Bradda Printing Services; 1993.

14. Hoffenberg EF, Ye J, Sun J, Ghomeshi HR, Salerno TA, Deslauriers R. Antegrade and retrograde continuous warm blood cardioplegia: a ${ }^{31} \mathrm{P}$ magnetic resonance study. Ann Thorac Surg 1995;60:1203-9.

15. Ally A, Park G. Rapid determination of creatine, phosphocreatine, purine bases, and nucleotides (ATP, ADP, AMP, GTP, GDP) in heart biopsies by gradient ion-pairing reversed-phase liquid chromatography. J Chromatogr 1992;575:19-27.

16. Somorjai RL, Morrow R, Deslauriers R. Quantification of in vivo
NMR spectra: a robust, interactive approach. Works in progress (Tenth Annual Meeting of the Society of Magnetic Resonance in Medicine); 1991 Aug 10-16; San Francisco. p. 1219.

17. Gadian DG, editor. Nuclear magnetic resonance and its applications to living systems. New York: Oxford University Press; 1982. p. 29-35.

18. Fisher LD, van Belle G, editors. Biostatistics. New York: John Wiley; 1993.

19. Lochner W, Arnold G, Muller-Rucholtz ER. Metabolism of the artificially arrested heart and of the gas perfused heart. Am J Cardiol 1968;22:299-311.

20. Hearse DJ, Stewart DA, Chain EB. Recovery from cardiac bypass and elective cardiac arrest: the metabolic consequences of various cardioplegic procedures in the isolated rat heart. Circ Res 1974;35:448-57.

21. Gundry SR, Wang N, Sciolaro CM, Van Arsdell GS, Razzouk AJ, Hill AC, et al. Uniformity of perfusion in all regions of the human heart by warm continuous retrograde cardioplegia. Ann Thorac Surg 1996;61:33-5.

22. Partington MT, Acar C, Buckberg GD, Julia P, Kofsky ER, Bugyi HI. Studies of retrograde cardioplegia. I. Capillary blood flow distribution to myocardium supplied by open and occluded arteries. J Thorac Cardiovasc Surg 1989;97:605-12.

23. Stirling MC, McClanahan TB, Schott RJ, Lynch MJ, Bolling SF, Kirsh MM, et al. Distribution of cardioplegic solution infused antegradely and retrogradely in normal canine hearts. J Thorac Cardiovasc Surg 1989;98:1066-76.

24. Solorzano J, Taitelbaum G, Chiu RC. Retrograde coronary sinus perfusion for myocardial protection during cardiopulmonary bypass. Ann Thorac Surg 1978;25:201-8.

25. Villanueva FS, Glasheen WP, Dent JM, Kaul S, Spotnitz WD. Discrepancy between capillary perfusion and myocardial cooling during retrograde cardioplegia: comparison with antegrade cardioplegia. Surg Forum 1992;213-5.

26. Tian G, Shen J, Su S, et al. R. Assessment of retrograde cardioplegia with magnetic resonance imaging and localized P-31 spectroscopy in isolated pig hearts. J Thorac Cardiovasc Surg 1997; 114:109-16.

27. Carrier M, Grégoire J, Khalil A, Thai P, Latour JG, Solymoss BC, et al. Myocardial distribution of cardioplegia administered by antegrade and retrograde routes to ischemic myocardium. Can J Surg 1997;40:108-13.

28. Yau TM, Weisel RD, Mickle DA, et al. Alternative techniques of cardioplegia. Circulation 1992;86(Suppl):II377-84.

29. Yau TM, Ikonomidis JS, Weisel RD, et al. Which technique of cardioplegia prevents ischemia? Ann Thorac Surg 1993;56:1020-8. 